\title{
LEAST-SQUARES APPROXIMATION BY ELEMENTS FROM MATRIX ORBITS ACHIEVED BY GRADIENT FLOWS ON COMPACT LIE GROUPS
}

\author{
CHI-KWONG LI, YIU-TUNG POON, AND THOMAS SCHULTE-HERBRÜGGEN
}

\begin{abstract}
Let $S(A)$ denote the orbit of a complex or real matrix $A$ under a certain equivalence relation such as unitary similarity, unitary equivalence, unitary congruences etc. Efficient gradient-flow algorithms are constructed to determine the best approximation of a given matrix $A_{0}$ by the sum of matrices in $S\left(A_{1}\right), \ldots, S\left(A_{N}\right)$ in the sense of finding the Euclidean least-squares distance$$
\min \left\{\left\|X_{1}+\cdots+X_{N}-A_{0}\right\|: X_{j} \in S\left(A_{j}\right), j=1, \ldots, N\right\} .
$$

Connections of the results to different pure and applied areas are discussed.
\end{abstract}

\section{INTRODUCTION}

Motivated by problems in pure and applied areas, there has been a great deal of interest in studying equivalence classes on matrices, say, under compact Lie group actions. For instance,

(a) the unitary (orthogonal) similarity orbit of a complex (real) square matrix $A$ is the set of matrices of the form $U A U^{*}$ for unitary (or real orthogonal) matrices $U$,

(b) the unitary (orthogonal) equivalence orbit of a complex (real) rectangular matrix $A$ is the set of matrices of the form $U A V$ for unitary (orthogonal) matrices $U, V$ of appropriate sizes,

(c) the unitary $t$-congruence orbit of a complex square matrix $A$ is the set of matrices of the form $U A U^{t}$ for unitary matrices $U$,

(d) the orthogonal similarity orbit of a complex square matrix $A$ is the set of matrices of the form $Q A Q^{t}$ for complex orthogonal matrices $Q$, i.e., $Q^{t} Q=I_{n}$,

(e) the similarity orbit of a square matrix $A$ is the set of matrices of the form $S A S^{-1}$ for invertible matrices $S$.

It is often useful to determine whether a matrix $A_{0}$ can be written as a sum of matrices from orbits $S\left(A_{1}\right), \ldots, S\left(A_{N}\right)$. Equivalently, one would like to know

Received by the editor September 15, 2008 and, in revised form, May 20, 2010.

2010 Mathematics Subject Classification. Primary 15A18, 15A60, 15A90; Secondary 37N30.

Key words and phrases. Complex Hermitian matrices, real symmetric matrices, eigenvalues, singular values, gradient flows.

The author is an honorary professor of the University of Hong Kong and an honorary professor of the Taiyuan University of Technology. His research was partially supported by USA NSF and the William and Mary Plumeri Award.

The second author's research was partially supported by USA NSF.

The third author is supported in part by the EU-programmes QAP, Q-ESSENCE and the exchange with COQUIT as well as by the excellence network of Bavaria through QCCC. 
whether

$$
S\left(A_{0}\right) \subseteq S\left(A_{1}\right)+\cdots+S\left(A_{N}\right) .
$$

For $N=1$, it reduces to the basic problem of checking whether $A_{0}$ is equivalent to $A_{1}$. In some cases, even this is nontrivial. For instance, it is not easy to check whether two $n \times n$ complex matrices are unitarily similar. For $N>1$, the problem is usually more involved. Even if there are theoretical results, it may not be easy to use them in practice or to check examples of matrices of moderate sizes. For instance, given $10 \times 10$ Hermitian matrices $A, B, C$, to conclude that $C=U A U^{*}+V B V^{*}$ for some unitary matrices $U$ and $V$, one needs to check thousands of inequalities involving the eigenvalues of $A, B$, and $C$; see Ref. 13. Therefore, one purpose of this paper is to set up a general framework to develop efficient computer algorithms and programs to solve such problems. In fact, we will treat the more general problem of finding the best approximation of a given matrix $A_{0}$ by the sum of matrices from matrix orbits $S\left(A_{1}\right), \ldots, S\left(A_{N}\right)$. In other words, for given matrices $A_{0}, A_{1}, \ldots, A_{N}$, we determine

$$
\min \left\{\left\|X_{1}+\cdots+X_{N}-A_{0}\right\|:\left(X_{1}, \ldots, X_{N}\right) \in S\left(A_{1}\right) \times \cdots \times S\left(A_{N}\right)\right\} .
$$

The results will be useful in solving numerical problems efficiently, and helpful in testing conjectures of theoretical development of the topics under consideration. As we will see in the following discussion, some numerical examples indeed lead to general theory; see Section 3.

We will consider different matrix orbits in the next few sections. In each case, we will mention the motivation of the problems and derive the gradient flows for the respective orbits, which will be used to design the algorithms and computer programs to solve the optimization problem. Note that we always consider the orbits of similarity $S A S^{-1}$ and equivalence $S A T$, where $\{S, T\}$ can be elements of any (semi)simple compact connected matrix Lie group, in particular, the special unitary group $S U(n)$ and subgroups thereof. Since these matrix Lie groups are compact, they are themselves smooth Riemannian manifolds $M$, which in turn implies they are endowed with a Riemannian metric induced by the non-degenerate Killing form related to a bi-invariant scalar product $\langle\cdot \mid \cdot\rangle_{x}$ on their tangent and cotangent spaces $T_{x} M$ and $T_{x}^{*} M$. The metric smoothly varies with $x \in M$ and allows for identifying the Fréchet differential in $T_{x}^{*} M$ with the gradient in $T_{x} M$. Moreover, in Riemannian manifolds the existence and convergence of gradient flows with appropriate discretization schemes are elaborated in detail in Ref. [32. In the present context, it is important to note that the subsequent gradient flows on the unitary congruence orbit and the unitary equivalence orbit are fundamental. The flows on compact connected subgroups of $S U(n)$ such as $S O(n)$ or $S U(2)^{\otimes m}$ (with $2^{m}=n$ ) can readily be derived from the flows on $S U(n)$ [31, 32. Furthermore, in each case, we will provide numerical examples to illustrate their efficiency and accuracy.

The situation in the general linear group $G L(N)$ and its subgroups that are not in the intersection with the unitary groups is entirely different: those groups are no longer compact, but only locally compact. For $G L(N)$ orbits we give an outlook with some analytical results in infima of Euclidean distances. Since locally compact Lie groups lack bi-invariant metrics on the tangent spaces to their orbit manifolds, they can only be endowed with left-invariant or right-invariant metrics. Moreover, the exponential map onto locally compact Lie groups is no longer geodesic as in 
the compact case. Consequently, one will have to devise other approximations to the respective geodesics than obtained by the (Riemannian) exponential. These numerics are thus a separate topic of current research and will therefore be pursued in a follow-up study.

With regard to notation, unless stated otherwise, the norm $\|A\|$ shall always be read as Frobenius norm $\|A\|_{2}:=\sqrt{\operatorname{tr}\left\{A^{*} A\right\}}$.

\section{UNITARY SIMILARITY ORBITS}

2.1. The Hermitian matrix case. For an $n \times n$ Hermitian matrix $A$, let $S(A)$ be the set of matrices unitarily similar to $A$. Then

$$
S(A)+S(B)=\{X+Y:(X, Y) \in S(A) \times S(B)\}
$$

is a union of unitary similarity orbits. Researchers have determined the necessary and sufficient conditions of $S(C)$ to be a subset of $S(A)+S(B)$ in terms of the eigenvalues of $A, B$ and $C ;$ 7, 8, 11, 13, 17, 19, 35, 36. In particular, suppose $A, B, C$ have eigenvalues

$$
a_{1} \geq \cdots \geq a_{n}, \quad b_{1} \geq \cdots \geq b_{n}, \quad \text { and } \quad c_{1} \geq \cdots \geq c_{n},
$$

respectively. Then $S(C) \subseteq S(A)+S(B)$ if and only if

$$
\sum_{j=1}^{n}\left(a_{j}+b_{j}-c_{j}\right)=0
$$

and a collection of inequalities in the form

$$
\sum_{r \in R} a_{r}+\sum_{s \in S} b_{s} \geq \sum_{t \in T} c_{t}
$$

for certain $m$-element subsets $R, S, T \subseteq\{1, \ldots, n\}$ with $1 \leq m<n$ determined by the Littlewood-Richardson rules; see Ref. [11, 13] for details. The study has connections to many different areas such as representation theory, algebraic geometry, and algebraic combinatorics, etc. Note that the relation between Horn's problem and the Littlewood-Richardson rules has recently also attracted attention in quantum information [9]. The set of inequalities in (2.2) grows exponentially with $n$. Therefore, it is not easy to check the conditions even for a moderate size problem, say, for $10 \times 10$ Hermitian matrices. As a matter of fact, the theory has been extended to determine whether $S\left(A_{0}\right)$ is a subset of $S\left(A_{1}\right)+\cdots+S\left(A_{N}\right)$ for given $n \times n$ Hermitian matrices $A_{0}, \ldots, A_{N}$, in terms of equality and linear inequalities of the eigenvalues of the given matrices. Of course, the number of inequalities involved are more numerous. There seems to be no efficient way of using these results in practice or testing numerical examples or conjecture in research.

It is interesting to note that by the saturation conjecture (theorem) (see Ref. [4] and its references), there exist Hermitian matrices with nonnegative integral eigenvalues $a_{1} \geq \cdots \geq a_{n}$, and $b_{1} \geq \cdots \geq b_{n}$ such that $A+B$ has nonnegative integral eigenvalues $c_{1} \geq \cdots \geq c_{n}$ if and only if the Young diagram corresponding to $\left(c_{1}, \ldots, c_{n}\right)$ can be obtained from those of $\left(a_{1}, \ldots, a_{n}\right)$ and $\left(b_{1}, \ldots, b_{n}\right)$. 
2.2. The general complex matrix case. Likewise, we study the problem

$$
\min \left\{\left\|\sum_{j=1}^{N} U_{j} A_{j} U_{j}^{*}-A_{0}\right\|: U_{1}, \ldots, U_{N} \in S U(n) \text { unitary }\right\}
$$

for general complex matrices $A_{0}, \ldots, A_{N}$. Even for $N=1$, the result is highly nontrivial. In theory, it is related to the problem of determining whether $A_{0}$ and $A_{1}$ are unitarily similar; see Ref. 33. Also, to determine

$$
\min \left\{\left\|U A U^{*}-C^{*}\right\|: U \text { unitary }\right\}
$$

for $A, C \in M_{n}$ leads to the study of the $C$-numerical range and the $C$-numerical radius of $A$ defined by

$$
W(C, A)=\left\{\operatorname{tr}\left(C U A U^{*}\right): U \in S U(n)\right\}
$$

and

$$
r(C, A)=\max \{|\mu|: \mu \in W(C, a)\} .
$$

The $C$-numerical radius is important in the study of unitary similarity invariant norms on $M_{n}$, i.e., norms $\nu$ satisfy $\nu\left(U X U^{*}\right)=\nu(X)$ for all $X, U \in M_{n}$ such that $U$ is unitary. For instance, it is known that for every unitary similarity invariant norm $\nu$ there is a compact subset $S$ of $M_{n}$ such that

$$
\nu(X)=\max \{r(C, X): C \in S\} .
$$

So, the $C$-numerical radii can be viewed as the building blocks of unitary similarity invariant norms. We refer readers to the survey [24] for further results on the $C$ numerical range and $C$-numerical radius. For applications of $C$-numerical ranges in quantum dynamics, see also Ref. [31]

For two matrices, one may study whether $C=U A U^{*}+V B V^{*}$ for a Hermitian $A$ and a skew-Hermitian $B$. In other words, we want to study whether a matrix can be written as the sum of a Hermitian matrix and a skew-Hermitian matrix with prescribed eigenvalues.

2.3. Sum of Hermitian and Skew-Hermitian matrices. For $C=U A U^{*}+$ $V B V^{*}$ with $A=A^{*}$ and $B=-B^{*}$, there are many known inequalities relating the eigenvalues of $A$ and $B$ to the eigenvalues and singular values of $C$; see Ref. [5] and the references therein. However, given $A=A^{*}$ and $B=-B^{*}$ with prescribed eigenvalues, there has been no known necessary and sufficient condition for the existence of $C$ with prescribed singular values satisfying $C=U A U^{*}+V B V^{*}$. Nevertheless, it is easy to solve the approximation problem

$$
\min \left\{\left\|U^{*} A U+V^{*} B V-C\right\|: U, V \text { unitary }\right\} .
$$

The following result actually holds for any unitarily invariant norm on $n \times n$ matrices using the same proof; see Ref. [26]. Furthermore, we can use this result to verify that our algorithm indeed yields an optimal solution; see Example 2 in Section 2.5.

Theorem 2.1. Let $\|\cdot\|$ be the Frobenius norm on $M_{n}$. Let $A, B, C \in M_{n}$ with $A=$ $A^{*}$ and $B=-B^{*}$. Moreover, let $a_{i}\left(\right.$ and $b_{i}$ ) denote the eigenvalues of $A$ (respectively, $-i B)$. Suppose $U, V \in M_{n}$ are unitary matrices such that $U \frac{1}{2}\left(C+C^{*}\right) U^{*}=$ $\operatorname{diag}\left(f_{1}, \ldots, f_{n}\right)$ with $f_{1} \geq \cdots \geq f_{n}$, and $V \frac{1}{2}\left(C-C^{*}\right) V^{*}=i \operatorname{diag}\left(g_{1}, \ldots, g_{n}\right)$ with $g_{1} \geq \cdots \geq g_{n}$. Suppose $A$ is unitarily similar to a diagonal matrix $A_{1}$ (respectively, $\left.A_{2}\right)$ with diagonal entries arranged in descending (respectively, ascending) order. 
Suppose $-i B$ is unitarily similar to a diagonal matrix $-i B_{1}$ (respectively, $-i B_{2}$ ) with diagonal entries arranged in descending (respectively, ascending) order. Then

$$
\begin{aligned}
\left\|U^{*} A_{1} U+V^{*} B_{1} V-C\right\|^{2} & =\sum_{j=1}^{n}\left(\left|f_{j}-a_{j}\right|^{2}+\left|g_{j}-b_{j}\right|^{2}\right), \\
\left\|U^{*} A_{2} U+V^{*} B_{2} V-C\right\|^{2} & =\sum_{j=1}^{n}\left(\left|f_{j}-a_{n-j+1}\right|^{2}+\left|g_{j}-b_{n-j+1}\right|^{2}\right),
\end{aligned}
$$

and for any unitary $X, Y \in M_{n}$,

$$
\left\|U^{*} A_{1} U+V^{*} B_{1} V-C\right\| \leq\left\|X^{*} A X+Y^{*} B Y-C\right\| \leq\left\|U^{*} A_{2} U+V^{*} B_{2} V-C\right\| .
$$

Proof. Let $F=\frac{1}{2}\left(C+C^{*}\right)$ and $G=\frac{-i}{2}\left(C-C^{*}\right)$. It is well known that

$$
\left\|F-U^{*} A_{1} U\right\| \leq\left\|F-X^{*} A X\right\| \leq\left\|F-U^{*} A_{2} U\right\|
$$

and

$$
\left\|G-V^{*} B_{1} V\right\| \leq\left\|G-Y^{*} B Y\right\| \leq\left\|G-V^{*} B_{2} V\right\|
$$

for any unitary $X, Y \in M_{n}$; see Ref. [26. Since $\|H+i K\|^{2}=\|H\|^{2}+\|K\|^{2}$ for any Hermitian $H, K \in M_{n}$, the results follow.

2.4. Deriving gradient flows on unitary similarity orbits. To begin with, we focus on the problem of approximating a given matrix $C$ using matrices from two unitary similarity orbits, i.e., finding

$$
\min \left\{\left\|U A U^{*}+V B V^{*}-C\right\|: U, V \in S U(n) \text { unitary }\right\} .
$$

For simplicity, here we describe the steepest descent method to search for unitary matrices $U_{0}, V_{0}$ attaining the optimum. Refined approaches like conjugate gradients, Jacobi-type or Newton-type methods may be implemented likewise; see for instance [32. As will be shown below, more than two unitary similarity orbits can be treated similarly. The basic idea is to improve the current unitary pair $\left(U_{k}, V_{k}\right)$ to $\left(U_{k+1}, V_{k+1}\right)$ so that

$$
\left\|U_{k+1} A U_{k+1}^{*}+V_{k+1} B V_{k+1}^{*}-C\right\|<\left\|U_{k} A U_{k}^{*}+V_{k} B V_{k}^{*}-C\right\|
$$

until the successive iterations differ only by a small tolerance, or the gradient (vide infra) vanishes. Further, to avoid pitfalls by local minima whenever the Euclidean distance cannot be made zero, we use a sufficiently large multitude of different random starting points $\left(U_{0}, V_{0}\right)$ for our algorithm. Needless to say, a positive matching result is constructive, while a negative result may be due to local minima. It is therefore important to use a sufficiently large set of initial conditions for confident conclusions in the negative case.

For a start, consider the least-squares minimization task

$$
\min _{U, V \in S U(n)}\left\|U A U^{*}+V B V^{*}-C\right\|_{2}^{2},
$$

which can be rewritten as

$$
\begin{aligned}
& \left\|U A U^{*}+V B V^{*}-C\right\|_{2}^{2} \\
& \quad=\left\|U A U^{*}+V B V^{*}\right\|_{2}^{2}+\|C\|_{2}^{2}-2 \operatorname{Re} \operatorname{tr}\left\{C^{*}\left(U A U^{*}+V B V^{*}\right)\right\} \\
& \quad=\|A\|_{2}^{2}+\|B\|_{2}^{2}+\|C\|_{2}^{2}-2 \operatorname{Re} \operatorname{tr}\left\{C^{*}\left(U A U^{*}+V B V^{*}\right)-U A U^{*} V B^{*} V^{*}\right\}
\end{aligned}
$$


and thus is equivalent to the maximization task

$$
\max _{U, V \in S U(n)} \operatorname{Retr}\left\{C^{*}\left(U A U^{*}+V B V^{*}\right)-U A U^{*} V B^{*} V^{*}\right\} .
$$

Therefore, we set

$$
f(U, V):=\operatorname{tr}\left\{\left(U A U^{*}+V B V^{*}\right) C^{*}-U A U^{*} V B^{*} V^{*}\right\}
$$

and $F(U, V):=\operatorname{Re} f(U, V)$. Then its Fréchet derivative $D_{U} f(U): T_{U} \mathcal{U} \rightarrow T_{f(U)} \mathcal{U}$ can be seen as a tangent map, where the elements of the tangent space $T_{U} \mathcal{U}$ to the Lie group of unitaries $\mathcal{U}=S U(n)$ or $U(n)$ at the point $U$ take the form $\Omega U$ with $\Omega=-\Omega^{*}$ being itself an element of the Lie algebra. The differential thus reads

$$
\begin{aligned}
D_{U} f(U, V)(\Omega U) & =\operatorname{tr}\left\{\left((\Omega U) A U^{*}+U A(\Omega U)^{*}\right)\left(C^{*}-V B^{*} V^{*}\right)\right\} \\
& =\operatorname{tr}\left\{\left((\Omega U) A U^{*}-U A U^{*}(\Omega U) U^{*}\right)\left(C^{*}-V B^{*} V^{*}\right)\right\} \\
& =\operatorname{tr}\left\{\left(A U^{*}\left(C^{*}-V B^{*} V^{*}\right)-U^{*}\left(C^{*}-V B^{*} V^{*}\right) U A U^{*}\right)(\Omega U)\right\}
\end{aligned}
$$

where we used the invariance of the trace under cyclic permutations as well as $(\Omega U)^{*}=-U^{*}(\Omega U) U^{*}$. Moreover, by identifying

$$
D_{U} f(U, V) \cdot(\Omega U)=\left\langle\operatorname{grad}_{U} f(U, V) \mid \Omega U\right\rangle=\operatorname{tr}\left\{\left(\operatorname{grad}_{U} f(U, V)\right)^{*} \Omega U\right\}
$$

one finds

$$
\begin{aligned}
\operatorname{grad}_{U} f(U, V) & =\left(C-V B V^{*}\right) U A^{*}-U A^{*} U^{*}\left(C-V B V^{*}\right) U \\
& =\left[\left(C-V B V^{*}\right), U A^{*} U^{*}\right] U .
\end{aligned}
$$

With $\left[X^{*}, Y\right]_{s}:=\frac{1}{2}\left(\left[X^{*}, Y\right]-\left[X^{*}, Y\right]^{*}\right)=\frac{1}{2}\left(\left[X^{*}, Y\right]+\left[X, Y^{*}\right]\right)$ as the skew-Hermitian part of the commutator one obtains for $F(U, V):=\operatorname{Re} f(U, V)$,

$$
\operatorname{grad}_{U} F(U, V)=\left[\left(C^{*}-V B^{*} V^{*}\right), U A U^{*}\right]_{s} U .
$$

Taking the respective Riemannian exponentials $\exp _{U}\left(\operatorname{grad}_{U} F(U, V)\right)$ as well as $\exp _{V}\left(\operatorname{grad}_{V} F(U, V)\right)$ thus gives the recursive gradient flows

$$
\begin{aligned}
U_{k+1} & =\exp \left\{-\alpha_{k}\left[U_{k} A U_{k}^{*},\left(C^{*}-V_{k} B^{*} V_{k}^{*}\right)\right]_{s}\right\} U_{k}, \\
V_{k+1} & =\exp \left\{-\beta_{k}\left[V_{k} B V_{k}^{*},\left(C^{*}-U_{k} A^{*} U_{k}^{*}\right)\right]_{s}\right\} V_{k},
\end{aligned}
$$

as discretized solutions of the coupled gradient system

$$
\dot{U}=\operatorname{grad}_{U} F(U, V) \text { and } \dot{V}=\operatorname{grad}_{V} F(U, V) .
$$

Conditions for convergence are described in detail in [16. For appropriate step sizes $\alpha_{k}, \beta_{k}$ see also Ref. [15].

Generalizing the findings from a sum of two orbits to higher sums of unitary orbits is straightforward: the problem

$$
\min \left\{\left\|\sum_{j=1}^{N} U_{j} A_{j} U_{j}^{*}-A_{0}\right\|: U_{1}, \ldots, U_{N} \in S U(n) \text { unitary }\right\}
$$

can be addressed by the system of coupled gradient flows $(j=1,2, \ldots, N)$

$$
U_{k+1}^{(j)}=\exp \left\{-\alpha_{k}^{(j)}\left[A_{k}^{(j)}, A_{0 j k}^{*}\right]_{s}\right\} U_{k}^{(j)}
$$

where for short we set $A_{k}^{(j)}:=U_{k}^{(j)} A_{j} U_{k}^{(j)^{*}}$ and $A_{0 j k}:=A_{0}-\sum_{\substack{\nu=1 \\ \nu \neq j}}^{N} A_{k}^{(\nu)}$.

These gradient flows follow the extension of the original idea on the orthogonal group [3, 16] to the unitary group [14, where now we have introduced a larger system of coupled flows. 


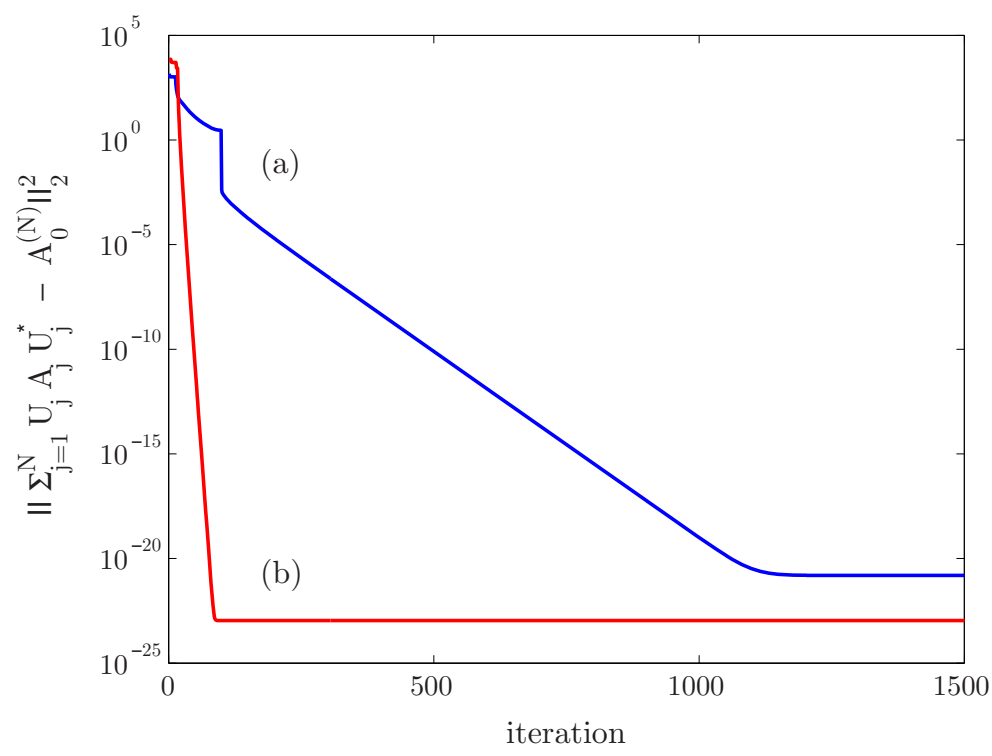

Figure 1. Coupled flows minimizing $\left\|\sum_{j=1}^{N} U_{j} A_{j} U_{j}^{*}-A_{0}^{(N)}\right\|_{2}^{2}$ with (a) $N=2$ and (b) $N=10$ for Example 1 below.

2.5. Numerical examples. Here we demonstrate gradient flows for minimizing $\left\|\sum_{j=1}^{N} U_{j} A_{j} U_{j}^{*}-A_{0}\right\|$ over the unitaries $U_{1}, \ldots, U_{N}$ for given Hermitian matrices $A_{0}, \ldots, A_{N}$.

Example 1. As a test case, consider the following examples for finding respective $U_{j} \in \mathbf{C}^{10 \times 10}$. For $j=1,2, \ldots, N$ choose a set of random unitaries $U_{j}^{(r)} \in$ $\mathbf{C}^{10 \times 10}$ distributed according to the Haar measure as recently described in 29] and define the diagonal matrices $A_{j}:=\operatorname{diag}(1,3,5, \ldots, 19)+\frac{j-1}{10} \mathbb{1}_{10}$ and $A_{0}^{(N)}:=$ $\operatorname{diag}\left(a_{1}, \ldots, a_{10}\right)$ where $a_{1}, a_{2}, \ldots, a_{10}$ are the eigenvalues of

$$
A_{0, N}^{\prime}:=\sum_{j=1}^{N} U_{j}^{(r)} A_{j} U_{j}^{(r)^{*}}
$$

(and $\mathbb{1}_{10}$ is the $10 \times 10$ identity matrix).

As shown in Figure 1, the gradient flow of (2.10) minimizes $\| \sum_{j=1}^{N} U_{j} A_{j} U_{j}^{*}-$ $A_{0}^{(N)} \|_{2}^{2}$ by driving it practically to zero. Note that in Figure 1(b) the combined flow on $N=10$ unitaries converges even faster than in Figure 1(a), where $N=2$ and the flow is more sensitive to saddle points as may be inferred from the jumps in trace (a).

Example 2. Let $A, B$ be Hermitian and $C$ arbitrary, e.g., $A=\left(\begin{array}{ccc}2 & 5 & 11 \\ 5 & 8 & 15 \\ 11 & 15 & 16\end{array}\right), B=$ $\left(\begin{array}{ccc}6 & 8 & 9 \\ 8 & 12 & 10 \\ 9 & 10 & 0\end{array}\right)$, and $C=\left(\begin{array}{ccc}1 & 1 & 3 \\ 6 & 9 & 3 \\ 8 & 9 & 2\end{array}\right)$. Thus $a:=\operatorname{eig}(A)=(-5.6674 ;-0.4830 ; 32.1504)$, $b:=\operatorname{eig}(B)=(-7.4816 ; 0.7123 ; 24.7693)$ and $f:=\operatorname{eig} \frac{1}{2}\left(C+C^{*}\right)=(-4.9555$; 
$-1.3888 ; 18.3443)$, while $g:=\operatorname{eig} \frac{-i}{2}\left(C-C^{*}\right)=(-4.6368 ; 0 ; 4.6368)$. By Theorem 2.1 one gets

$$
\begin{aligned}
\Delta: & =\min _{U, V \in S U(3)}\left\|U A U^{*}+i V B V^{*}-C\right\|_{2}^{2} \\
& =(a-f)^{*}(a-f)+(b-g)^{*}(b-g) \\
& =605.8521 \ldots .
\end{aligned}
$$

More precisely, $\Delta=605.852131091^{\prime} 3004$, while 100 runs of the flow with independent random initial conditions give a mean \pm rmsd of $\bar{\Delta}=605.852131091^{\prime} 3570 \pm$ $1.13 \cdot 10^{-10}$.

\section{UNITARY EQUIVALENCE}

In this section, we study

$\min \left\{\left\|\sum_{j=1}^{N} U_{j} A_{j} V_{j}-A_{0}\right\|: U_{1}, \ldots, U_{N} \in U(n) \quad\right.$ and $\quad V_{1}, \ldots, V_{N} \in U(m)$ unitary $\}$

for rectangular matrices $A_{0}, \ldots, A_{N}$. By the result of O'Shea and Sjamaar [34,

$$
\min \left\|\sum_{j=1}^{N} U_{j} A_{j} V_{j}-A_{0}\right\|=0
$$

if and only if

$$
\min \left\|\sum_{j=1}^{N} W_{j}^{*} \tilde{A}_{j} W_{j}-\tilde{A}_{0}\right\|=0
$$

where

$$
\tilde{A}_{j}=\left(\begin{array}{cc}
0 & A_{j} \\
A_{j}^{*} & 0
\end{array}\right) \quad \text { for } j=0,1, \ldots, N .
$$

Thus, by the results concerning unitary similarity orbits (see Section 2),

$$
\min \left\{\left\|A_{0}-\sum_{j=1}^{N} U_{j} A_{j} V_{j}\right\|: U_{1}, \ldots, U_{N} ; V_{1}, \ldots, V_{N} \text { unitary }\right\}=0
$$

if and only if the singular values of $A_{0}, A_{1}, \ldots, A_{N}$ satisfy a certain set of linear inequalities. Clearly, $\min \{\|A-U B V\|: U, V$ unitary $\}=0$ if and only if $A$ and $B$ have the same singular values. In general, it is interesting to check whether

$$
\sqrt{2} \min \left\|\sum_{j=1}^{N} U_{j} A_{j} V_{j}-A_{0}\right\|=\min \left\|\sum_{j=1}^{N} W_{j}^{*} \tilde{A}_{j} W_{j}-\tilde{A}_{0}\right\|=0 .
$$

In computer experiments (see Example 6 in Section 3), we observe that (3.1) always holds if $A_{0}, A_{1}, \ldots, A_{N}$ are randomly generated matrices generated by MATLAB. We explain this phenomenon in the following. We begin with a simple observation.

Lemma 3.1. Suppose $a_{0}, a_{1}, \ldots, a_{N} \in(0, \infty)$. The following are equivalent.

(a) There are complex units $e^{i t_{1}}, \ldots, e^{i t_{N}}$ such that $a_{0}-\sum_{j=1}^{N} a_{j} e^{i t_{j}}=0$.

(b) There is an $N+1$ side convex polygon whose sides have lengths $a_{0}, \ldots, a_{N}$.

(c) $\sum_{j=0}^{N} a_{j}-2 a_{k} \geq 0$ for all $k=0,1, \ldots, N$. 
From this observation, one easily gets the following condition related to the equality (3.1).

Proposition 3.2. Let $A_{j}=\operatorname{diag}\left(a_{1 j}, \ldots, a_{n j}\right)$ be nonnegative diagonal matrices for $j=0,1, \ldots, N$, and let $v_{j}=\left(a_{1 j}, \ldots, a_{n j}\right)^{t}$. Then there exist permutation matrices $P_{1}, \ldots, P_{N}$ and diagonal unitary matrices $D_{1}, \ldots, D_{N}$ such that

$$
A_{0}=\sum_{j=1}^{N} D_{j} P_{j} A_{j} P_{j}^{t}
$$

if and only if the entries of each row of the matrix

$$
\left[v_{0}\left|P_{1} v_{1}\right| \cdots \mid P_{N} v_{N}\right]
$$

correspond to the sides of an $N+1$ side convex polygon.

If one examines the singular values of an $n \times n$ "random matrix" generated by MATLAB, we see that there is always a dominant singular value of size about $n / 2$, and the other singular values range from 0 to $1.5 n$ in a rather systematic pattern. So, it is often possible to apply Proposition 3.2 to get equality (3.1) if $A_{0}, \ldots, A_{N}$ are "random matrices" generated by MATLAB for $N \geq 2$ as will be made more precise in Example 6 (vide infra).

In contrast, for general matrices, it is easy to construct $A_{0}, A_{1}, \ldots, A_{N}$ such that (3.1) fails.

Example 3. Let $A_{0}=\operatorname{diag}\left(N^{2}, N+1\right) \oplus \mathbb{O}_{n-2}$ and $A_{j}=\operatorname{diag}(N, 1) \oplus \mathbb{O}_{n-2}$ for $j=1, \ldots, N$. Then clearly (3.1) does not apply, because

$$
\sum_{j=1}^{n} s_{j}\left(A_{0}\right)>\sum_{i=1}^{N} \sum_{j=1}^{n} s_{j}\left(A_{j}\right)
$$

Recall that the Ky Fan $k$-norm of a matrix $A \in M_{n}$ is defined as $\|A\|_{k}=$ $\sum_{j=1}^{k} s_{j}(A)$, and a norm $\|\cdot\|$ on $M_{n}$ is unitarily invariant if $\|A\|=\|U A V\|$ for all $A \in M_{n}$ and unitary $U, V \in M_{n}$. By the Ky Fan dominance theorem, two matrices $A, B \in M_{n}$ satisfy $\|A\|_{k} \leq\|B\|_{k}$ for $k=1, \ldots, n$ if and only if $\|A\| \leq\|B\|$ for all unitarily invariant norms $\|\cdot\|$. In view of this example, we have the following result.

Proposition 3.3. Suppose $A_{0}, A_{1}, \ldots, A_{N} \in M_{n}$ satisfy (3.1). Then for all unitarily invariant norms,

$$
2\left\|A_{i}\right\| \leq \sum_{j=0}^{N}\left\|A_{j}\right\|, \quad i=0,1, \ldots, N,
$$

and equivalently, for $k=1, \ldots, n$,

$$
2\left\|A_{i}\right\|_{k} \leq \sum_{j=0}^{N}\left\|A_{j}\right\|_{k}, \quad i=0,1, \ldots, N .
$$

Moreover, if there is $k$ such that equality (3.2) holds, then (3.1) holds if and only if $A_{j}$ is unitarily similar to $B_{j} \oplus C_{j}$ with $B_{j} \in M_{k}$ for $j=0, \ldots, N$ such that

$$
\min \left\{\left\|B_{0}-\sum_{j=1}^{N} U_{j} B_{j} V_{j}\right\|: U_{1}, \ldots, U_{N}, V_{1}, \ldots, V_{N} \in M_{k} \text { are unitary }\right\}=0
$$


and

$$
\min \left\{\left\|C_{0}-\sum_{j=1}^{N} X_{j} C_{j} Y_{j}\right\|: X_{1}, \ldots, X_{N}, Y_{1}, \ldots, Y_{N} \in M_{n-k} \text { are unitary }\right\}=0 .
$$

It would be nice if one can get (3.1) by checking the relatively easy condition (3.2). Unfortunately, the following example shows that it is not true.

Example 4. Let $A_{0}=\operatorname{diag}(14,2), A_{1}=\operatorname{diag}(8,0), A_{2}=\operatorname{diag}(7,4)$. Then (3.2) is satisfied for all $k \geq 1$ but by the result in [25],

$$
\operatorname{diag}\left(U_{1} A_{1} V_{1}+U_{2} A_{2} V_{2}\right) \neq(14,2)
$$

for all unitaries $U_{i}, V_{j}$.

3.1. Deriving gradient flows on unitary equivalence orbits. For minimizing $\|U A V-C\|_{2}^{2}$ one has to maximize

$$
F(U, V):=\operatorname{Re} \operatorname{tr}\left\{U A V C^{*}\right\}=\frac{1}{2} \operatorname{tr}\left\{U A V C^{*}+\left(U A V C^{*}\right)^{*}\right\} .
$$

By the same arguments as before, from its Fréchet differential

$$
\begin{aligned}
D_{U} F(U, V)(\Omega U) & =\frac{1}{2} \operatorname{tr}\left\{(\Omega U) A V C^{*}-C V^{*} A^{*} U^{*}(\Omega U) U^{*}\right\} \\
& =\frac{1}{2} \operatorname{tr}\left\{\left(A V C^{*}-U^{*} C V^{*} A^{*} U^{*}\right)(\Omega U)\right\}
\end{aligned}
$$

one obtains the gradient-where henceforth we keep writing $(\cdot)_{s}$ for the skewHermitian part

$$
\operatorname{grad}_{U} F(U, V)=\frac{1}{2}\left(A V C^{*}-U^{*} C V^{*} A^{*} U^{*}\right)^{*}=-\left(U A V C^{*}\right)_{s} U .
$$

An analogous result follows for $\operatorname{grad}_{V} F(U, V)$. Taking again the respective Riemannian exponentials leads to the recursive scheme

$$
\begin{aligned}
U_{k+1} & =\exp \left\{-\alpha_{k}\left(U_{k} A V_{k} C^{*}\right)_{s}\right\} U_{k} \\
V_{k+1} & =\exp \left\{-\beta_{k}\left(V_{k} C^{*} U_{k} A\right)_{s}\right\} V_{k},
\end{aligned}
$$

which also can be used, e.g., for a singular-value decomposition of $A$ by choosing $C$ real diagonal.

Likewise, minimizing $\|U A V+X B Y-C\|_{2}^{2}$ by maximizing the trace expression $\operatorname{Re} \operatorname{tr}\left\{U A V(C-X B Y)^{*}+X B Y C^{*}\right\}$ translates into the same flows when substituting $C \mapsto\left(C-X_{k} B Y_{k}\right)$ with analogous recursions for $X_{k+1}$ and $Y_{k+1}$. Along these lines, it is straightforward to address the general task

$\min \left\{\left\|\sum_{j=1}^{N} U_{j} A_{j} V_{j}-A_{0}\right\|: U_{1}, \ldots, U_{N} \in U(n) \quad\right.$ and $\quad V_{1}, \ldots, V_{N} \in U(m)$ unitary $\}$ with rectangular matrices $A_{0}, \ldots, A_{N}$ by a system of $2 N$ coupled gradient flows $(j=1,2, \ldots, N)$,

$$
\begin{aligned}
& U_{k+1}^{(j)}=\exp \left\{-\alpha_{k}^{(j)}\left(U_{k}^{(j)} A_{j} V_{k}^{(j)} A_{0 j k}^{*}\right)_{s}\right\} U_{k}^{(j)}, \\
& V_{k+1}^{(j)}=\exp \left\{-\beta_{k}^{(j)}\left(V_{k}^{(j)} A_{0 j k}^{*} U_{k}^{(j)} A_{j}\right)_{s}\right\} V_{k}^{(j)},
\end{aligned}
$$

where we use the shorthand $A_{0 j k}:=A_{0}-\sum_{\substack{\nu=1 \\ \nu \neq j}}^{N} U_{k}^{(\nu)} A_{\nu} V_{k}^{(\nu)}$.

Clearly, the set of unitaries $\left\{\tilde{U}_{j}\right\} \cup\left\{\tilde{V}_{j}\right\}$ achieving a global minimum is not unique. Without loss of generality, assume that for $j=0,1,2, \ldots, N$, the $A_{j} \in \mathbf{C}^{n \times m}$ 
(chosen with $n \leq m$ ) are initially given each in their singular-value decomposition $\Sigma_{j}=\left[\Lambda_{j} \mid \mathbb{O}_{n,(m-n)}\right] \in \mathbf{R}^{n \times m}$ (where the diagonal $\Lambda_{j}$ consists of the ordered singular values of the respective $\left.A_{j}\right)$. With the shorthand notation $\mathbf{U}:=\left(U_{1}, U_{2}, \ldots, U_{N}\right)$ ( $\mathbf{V}$ analogously) the minimum in (3.3) is degenerate and takes the form 1

$$
F_{\min }(\mathbf{U}, \mathbf{V})=F(\tilde{\mathbf{U}}, \tilde{\mathbf{V}})=\left\|\sum_{j=1}^{N} \tilde{U}_{j} \Sigma_{j} \tilde{V}_{j}-\Sigma_{0}\right\|=\left\|\sum_{j=1}^{N} \tilde{A}_{j}-\Sigma_{0}\right\| .
$$

The $\left\{\tilde{U}_{j}\right\}$ are only determined up to their respective right diagonal factors reading $\tilde{D}_{j}:=\operatorname{diag}\left(e^{i \phi_{1}^{(j)}}, e^{i \phi_{2}^{(j)}}, \ldots, e^{i \phi_{n}^{(j)}}\right)$ with free independent $\phi_{\ell}^{(j)} \in[0,2 \pi)$ for all the $j=1,2, \ldots, N$ and $\ell=1,2, \ldots, n$ such that $\tilde{U}_{j} \tilde{D}_{j} \Sigma_{j} \tilde{V}_{j}^{\prime}=\tilde{A}_{j}$. Given $\tilde{D}_{j}$, the $\tilde{V}_{j}^{\prime}$ are also not unique unless $\operatorname{rank} A_{j}=n=m$, in which case $\tilde{V}_{j}^{\prime}=\Sigma_{j}^{-1} \tilde{D}_{j}^{*} \tilde{U}_{j}^{*} \tilde{A}_{j}$. As usual, further degrees of freedom emerge for degenerate singular values or for singular values being zero (see, e.g., Lem. 7.3.1 and Thm. 7.3.5 in [18).

Another symmetry arises if there exist nontrivial pairs of unitaries $\left(U_{s}, V_{s}\right) \in$ $U(n) \times U(m)$ stabilizing $A_{0}$ in the sense that $U_{s} A_{0} V_{s}=A_{0}$. Then the $\left\{U_{s}\right\}$ and $\left\{V_{s}\right\}$ form subgroups $\mathcal{U}_{s} \subseteq U(n)$ and $\mathcal{V}_{s} \subseteq U(m)$. By unitary invariance of the Frobenius norm, the union of joint left and right $\operatorname{cosets} \mathcal{U}_{s}\left\{\tilde{U}_{j}\right\} \cup\left\{\tilde{V}_{j}\right\} \mathcal{V}_{s}$ give the same minimum as $\left\{\tilde{U}_{j}\right\} \cup\left\{\tilde{V}_{j}\right\}$ because for all $U_{s} \in \mathcal{U}_{s}$ and $V_{s} \in \mathcal{V}_{s}$,

$$
F_{\min }(\mathbf{U}, \mathbf{V})=\left\|\sum_{j=1}^{N} \tilde{U}_{j} A_{j} \tilde{V}_{j}-A_{0}\right\|=\left\|U_{s}\left(\sum_{j=1}^{N} \tilde{U}_{j} A_{j} \tilde{V}_{j}\right) V_{s}-A_{0}\right\|,
$$

where again the $V_{s}$ are uniquely determined by the $U_{s}$ only if $\operatorname{rank} A_{0}=n=m$. An analogous argument covers stabilizing orbits of the total sum (as well as potential invariances of partial sums with $j$ ranging over proper subsets of $\{1,2, \ldots, N\}$ ).

These degeneracies of the minimium $F_{\min }(\mathbf{U}, \mathbf{V})$ with respect to continuous degrees of freedom in the critical $\left\{\tilde{U}_{j}\right\} \cup\left\{\tilde{V}_{j}\right\}$ may contribute to the favorable convergence properties of our gradient-flow algorithms: envisaged as a landscape, $F(\mathbf{U}, \mathbf{V})$ shows critical valleys rather than isolated minima.

3.2. Numerical examples. Using the flows from Section 3.1, here we study

$\min \left\{\left\|\sum_{j=1}^{N} U_{j} A_{j} V_{j}-A_{0}\right\|: U_{1}, \ldots, U_{N} \in U(n) \quad\right.$ and $\quad V_{1}, \ldots, V_{N} \in U(m)$ unitary $\}$ for rectangular matrices $A_{0}, \ldots, A_{N}$.

Example 5. As an example of rectangular $A_{j} \in \mathbf{C}^{10 \times 15}$, consider the analogous flows. In order to obtain $U_{j} \in \mathbf{C}^{10 \times 10}$ and $V_{j} \in \mathbf{C}^{15 \times 15}$ for $j=1,2, \ldots, N$ choose a set of random unitary pairs $\left(U_{j}^{(r)}, V_{j}^{(r)}\right) \in \mathbf{C}^{10 \times 10} \times \mathbf{C}^{15 \times 15}$ and define

$A_{j}:=\left[\operatorname{diag}(1,3,5, \ldots, 19)+\frac{j-1}{10} \mathbb{1}_{10} \mid \mathbb{O}_{10,5}\right]$ and $A_{0}^{(N)}:=\left[\operatorname{diag}\left(s_{1}, \ldots, s_{10}\right) \mid \mathbb{O}_{10,5}\right]$ where $s_{1}, s_{2}, \ldots, s_{10}$ are now the singular values of $A_{0, N}^{\prime}:=\sum_{j=1}^{N} U_{j}^{(r)} A_{j} V_{j}^{(r)}$ and $\mathbb{O}_{10,5}$ is the $10 \times 5$ zero-matrix.

\footnotetext{
${ }^{1}$ With slight abuse of language, here $F(\mathbf{U}, \mathbf{V}):=\left\|\sum_{j=1}^{N} U_{j} A_{j} V_{j}-A_{0}\right\|$, which equals previous definitions up to a constant.
} 


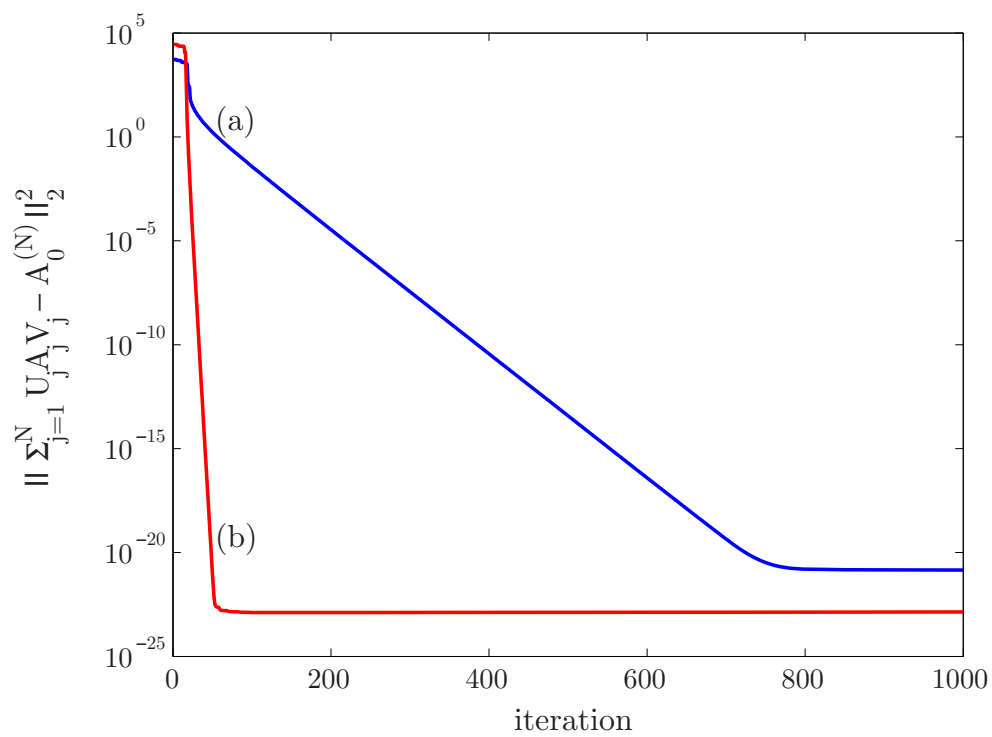

Figure 2. Coupled flows minimizing $\left\|\sum_{j=1}^{N} U_{j} A_{j} V_{j}-A_{0}^{(N)}\right\|_{2}^{2}$ with (a) $N=2$ and (b) $N=10$ for Example 5. The $A_{j} \in \mathbf{C}^{10 \times 15}$ are rectangular so that $U_{j} \in \mathbf{C}^{10 \times 10}$ and $V_{j} \in \mathbf{C}^{15 \times 15}$.

Figure 2 shows how the coupled gradient flow minimizes $\left\|\sum_{j=1}^{N} U_{j} A_{j} V_{j}-A_{0}^{(N)}\right\|_{2}^{2}$ by driving it practically to zero. Again the combined flow on $N=10$ unitary pairs (Figure 2(b)) converges faster than the one for $N=2$ unitary pairs given in Figure 2(a).

3.2.1. Observation Concerning Sums of Unitary Equivalence Orbits. Let $A_{j}(j=$ $0,1,2, \ldots, N)$ denote a series of complex $n \times m$ matrices each with random entries of the following types:

(a') real and imaginary parts of all the $a_{i k}^{(j)}$ are independently distributed by the standard normal Gaussian distribution with zero mean and variance unity (in MATLAB: $\operatorname{randn}(n, m)$ );

(a) same as (a'), but with final normalization to $\left\|A_{j}\right\|_{2}=1$.

(b') real and imaginary parts of all the $a_{i k}^{(j)}$ are independently uniformly distributed in the intervals $[0,1]$ (in MATLAB: $\operatorname{rand}(\mathrm{n}, \mathrm{m})$ );

(b) same as (b'), but with final normalization to $\left\|A_{j}\right\|_{2}=1$.

Let $\mathcal{O}_{U V}(X):=\{U X V: U, V$ independent unitary $\}$ denote the unitary equivalence orbit of $X$. For each of the above four instances of randomization the following observation holds: $A_{0}$ is typically distant from a single equivalence orbit of another random matrix $A_{1}$ of the same type since generically $A_{0}$ and $A_{1}$ clearly do not share the same singular values. However, in all of the four instances of randomization, such an $A_{0}$ is typically an element of the sum of two or more unitary equivalence 

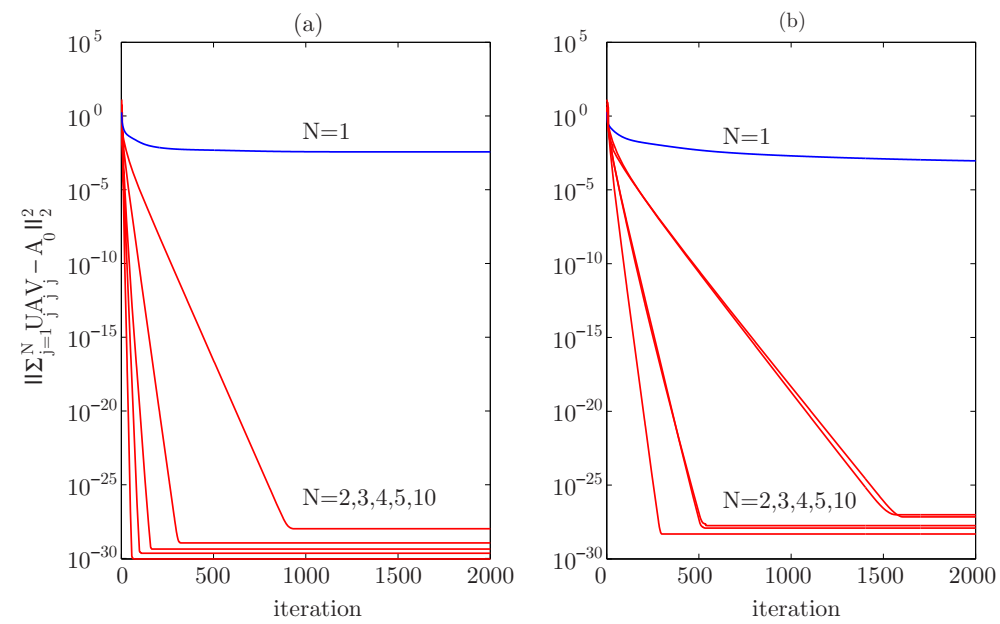

Figure 3. Typically, as illustrated in Example 6 a complex matrix $A_{0} \in \mathbf{C}^{10 \times 10}$ taken randomly as in the schemes (a) and (b) in 3.2.1 is distant from a single $(N=1)$ equivalence orbit of another random matrix $U A_{1} V$ (upper traces), yet it is an element of the sum of $N=2,3,4,5,10$ equivalence orbits of several independent random matrices as in the lower traces $\left\|\sum_{j=1}^{N} U_{j} A_{j} V_{j}-A_{0}\right\|_{2}^{2} \rightarrow 0$.

orbits of independent random matrices of the same kind, sd2

$$
A_{0} \notin \mathcal{O}_{U_{1} V_{1}}\left(A_{1}\right) \quad \text { while } \quad A_{0} \in \sum_{j=1}^{N} \mathcal{O}_{U_{j} V_{j}}\left(A_{j}\right) \quad \text { for } N \geq 2 .
$$

Interestingly, the findings hold independent of the dimensions and include rectangular matrices as well as square matrices. This is illustrated in Figure 3 for the above randomization scenarios (a) and (b) by numerical examples with $10 \times 10$ complex matrices thus underscoring Proposition 3.2.

Example 6. For complex matrices $A_{j} \in \mathbf{C}^{10 \times 10}$ with the real and imaginary parts of each element taken randomly from (a) the standard normal distribution with zero mean, variance unity and normalized to $\left\|A_{j}\right\|_{2}=1$ or taken randomly from (b) a uniform distribution over the intervals $[0,1]$ and again normalized one finds: such a random $A_{0}$ is typically an element of the sum of $N=2,3,4,5,10$ equivalence orbits $\sum_{j=1}^{N} U_{j} A_{j} V_{j}$, while it is typically not an element of a single $(N=1)$ such equivalence orbit. This is shown in Figure 3 (a) and (b); qualitatively analogous results are obtained without the normalization $\left\|A_{j}\right\|_{2}=1$ thus corresponding to the scenarios (a') and (b') in 3.2.1 (not shown).

In contrast, preliminary results indicate that the situation is more subtle for the respective Hermitian parts of such types of random matrices $H\left(A_{j}\right):=\frac{1}{2}\left(A_{j}+A_{j}^{*}\right)$

\footnotetext{
${ }^{2}$ Note that as mentioned earlier in the context of Proposition 3.2, it is easy to construct sets of matrices violating (3.6), however these sets are not typical with respect to the random distributions discussed here.
} 
and their relation to the unitary similarity orbits $\mathcal{O}_{U_{j}}\left(H\left(A_{j}\right)\right):=\left\{U_{j} H\left(A_{j}\right) U_{j}^{*}\right.$ : $U_{j}$ unitary $\}$. Explorative findings, again for $A_{j} \in \mathbf{C}^{10 \times 10}$, suggest that typically

$$
H\left(A_{0}\right) \notin \sum_{j=1}^{N} \mathcal{O}_{U_{j}}\left(H\left(A_{j}\right)\right),
$$

unless the real and imaginary parts of the matrix elements in $A_{j}$ are again taken randomly from either a uniform distribution over the interval $[0,1]$ or the standard normal distribution, where in either case the following normalization conditions have to be fulfilled as well: normalize to $\operatorname{tr} A_{0}=1$ as well as to $\operatorname{tr} A_{j}=1 / N$ for all the $j=1,2, \ldots, N$. Also, the number of similarity orbits in the sum has to be sufficiently large (here $N \simeq 10$ ) in order to violate (3.7) in more than $30 \%$ of the instances. For the context of completely positive maps (a.k.a. quantum channels) [6. 20, the above normalizations remotely resemble a density operator (here $A_{0}$ ) and, more loosely, a quantum map with Kraus rank $N$ (here $\sum_{j=1}^{N} \mathcal{O}_{U_{j}}\left(H\left(A_{j}\right)\right.$ ) with identical $A_{j}$ for all $\left.j=1,2, \ldots, N\right)$. Thus these preliminary findings will be further pursued elsewhere. (Note that in quantum physics for $\rho$ to be a density operator, $\rho$ also has to be positive semidefinite beyond fulfilling $\operatorname{tr} \rho=1$.)

\section{UNITARY $t$-CONGRUENCE}

In this section, we consider

$$
\min \left\{\left\|\sum_{j=1}^{N} U_{j} A_{j} U_{j}^{t}-A_{0}\right\|: U_{1}, \ldots, U_{N} \in U(n) \text { unitary }\right\}
$$

for given matrices $A_{0}, A_{1}, \ldots, A_{N}$. Sometimes, we can focus on special classes of matrices such as symmetric matrices or skew-symmetric matrices. For symmetric matrices or skew-symmetric matrices, the minimization problem

$$
\min \left\{\left\|U A U^{t}-A_{0}\right\|: U \text { unitary }\right\}
$$

has an analytic solution; see Ref. 28. The problem is entirely open even if $N=2$. Therefore, a computer algorithm will be most helpful in the theoretical development. One may also consider whether we can have $U A U^{t}+V B V^{t}=C$ for a symmetric $A$ and a skew-symmetric $B$. In other words, we want to know whether one can write $C$ as the sum of symmetric and skew-symmetric matrices with prescribed singular values. Of course, the problem for general matrices $A, B$ and $C$ is even more challenging, and that is what we pursue by the numerical methods developed in the next paragraph.

4.1. Gradient flows on unitary $t$-congruence orbits. Again, the minimization task

$$
\min _{U, V \in U(n)}\left\|U A U^{t}+V B V^{t}-C\right\|_{2}^{2}
$$

translates via

$$
\begin{aligned}
& \left\|U A U^{t}+V B V^{t}-C\right\|_{2}^{2} \\
& \quad=\|A\|_{2}^{2}+\|B\|_{2}^{2}+\|C\|_{2}^{2}-2 \operatorname{Retr}\left\{C^{*}\left(U A U^{t}+V B V^{t}\right)-U A U^{t} \bar{V} B^{*} V^{*}\right\}
\end{aligned}
$$

into maximizing the function

$$
F(U, V):=\operatorname{Re} f(U, V):=\operatorname{Retr}\left\{\left(U A U^{t}+V B V^{t}\right) C^{*}-U A U^{t} \bar{V} B^{*} V^{*}\right\}
$$


where the differential reads (by virtue of the shorthand $\tilde{C}:=C^{*}-\bar{V} B^{*} V^{*}$ )

$$
\begin{aligned}
D_{U} f(U, V)(\Omega U) & =\operatorname{tr}\left\{\left((\Omega U) A U^{t}+U A(\Omega U)^{t}\right)\left(C^{*}-\bar{V} B^{*} V^{*}\right)\right\} \\
& =\operatorname{tr}\left\{(\Omega U) A U^{t} \tilde{C}\right\}+\operatorname{tr}\left\{\left(U A(\Omega U)^{t} \tilde{C}\right)^{t}\right\} \\
& =\operatorname{tr}\left\{\left(A U^{t} \tilde{C}+A^{t} U^{t} \tilde{C}^{t}\right)(\Omega U)\right\} .
\end{aligned}
$$

By identifying $\left.D_{U} f(U, V) \cdot(\Omega U)=\left\langle\operatorname{grad}_{U} f(U, V) \mid \Omega U\right\rangle=\operatorname{tr}\left\{\operatorname{grad}_{U} f(U, V)\right)^{*} \Omega U\right\}$ one finds

$$
\operatorname{grad}_{U} f(U, V)=\left(U A U^{t} \tilde{C}+U A^{t} U^{t} \tilde{C}^{t}\right)^{*} U
$$

so as to obtain for $F(U, V):=\operatorname{Re} f(U, V)$,

$$
\operatorname{grad}_{U} F(U, V)=-\left(U A U^{t} \tilde{C}+U A^{t} U^{t} \tilde{C}^{t}\right)_{s} U .
$$

Again, taking the respective Riemannian exponentials $\exp _{U}\left(\operatorname{grad}_{U} F(U, V)\right)$ and $\exp _{V}\left(\operatorname{grad}_{V} F(U, V)\right)$ thus gives the slightly lengthy formula

$$
U_{k+1}=\exp \left\{-\alpha_{k}\left(U_{k} A U_{k}^{t}\left(C^{*}-\bar{V}_{k} B^{*} V_{k}^{*}\right)+U_{k} A^{t} U_{k}^{t}\left(C^{*}-\bar{V}_{k} B^{*} V_{k}^{*}\right)^{t}\right)_{s}\right\} U_{k},
$$

and an analogous equation for $V_{k+1}$ by substituting $V$ for $U$ and $B$ for $A$,as discretized solutions of the coupled gradient system

$$
\dot{U}=\operatorname{grad}_{U} F(U, V) \text { and } \dot{V}=\operatorname{grad}_{V} F(U, V) .
$$

Likewise, for higher sums of $t$-congruence orbits one finds

$$
\min \left\{\left\|\sum_{j=1}^{N} U_{j} A_{j} U_{j}^{t}-A_{0}\right\|: U_{1}, \ldots, U_{N} \in U(n) \text { unitary }\right\}
$$

to be solved by the coupled system of flows $(j=1,2, \ldots, N)$

$$
U_{k+1}^{(j)}=\exp \left\{-\alpha_{k}^{(j)}\left(A_{k}^{(j)} A_{0 j k}^{*}+\left(A_{0 j k}^{*} A_{k}^{(j)}\right)^{t}\right)_{s}\right\} U_{k}^{(j)},
$$

where for short we set $A_{k}^{(j)}:=U_{k}^{(j)} A_{j} U_{k}^{t(j)}$ and $A_{0 j k}:=A_{0}-\sum_{\substack{\nu=1 \\ \nu \neq j}}^{N} A_{k}^{(\nu)}$.

\section{Outlook: Noncompact Groups}

For orbits $S(A)$ of matrices $A$ under the action of noncompact groups, there are usually no good results for the supremum or the infimum of $\left\|X_{0}-\sum_{j=1}^{N} X_{j}\right\|$ with $X_{j} \in S\left(A_{j}\right)$ for $j=0,1, \ldots, N$ and given matrices $A_{0}, \ldots, A_{N}$.

For example, for the invertible congruence orbit of $A \in M_{n}$,

$$
S(A)=\left\{S^{*} A S: S \in M_{n} \text { is invertible }\right\},
$$

we can let $S=r \mathbb{1}$. Then

$$
\left\|S^{*} A_{0} S-\sum_{j=1}^{N} S^{*} A_{j} S\right\|
$$

converges to 0 or $\infty$ depending on $r \rightarrow 0$ or $r \rightarrow \infty$.

Similarly, the same problems occur for the equivalence orbit of $A \in M_{n}$ :

$$
S(A)=\left\{S A T: S, T \in M_{n} \text { are invertible }\right\} .
$$

For the similarity orbits, we have the following. 
Proposition 5.1. Suppose not all the matrices $A_{0}, \ldots, A_{N}$ are scalar. Then

$$
\sup \left\|A_{0}-\sum_{j=1}^{N} S_{j}^{-1} A_{j} S_{j}\right\|=\infty .
$$

Proof. Suppose one of the matrices, say, $A_{i}$ is nonscalar. Then there is $S_{j}$ such that $S_{j}^{-1} A_{i} S_{j}$ is in lower triangular form with the $(2,1)$ entry equal to 1 , and there are invertible matrices $S_{j}$ such that $S_{j}^{-1} A_{j} S_{j}$ is in upper triangular form for other $j$. Let $D_{r}=\operatorname{diag}(r, 1,1, \ldots, 1)$. Then the sequence

$$
\left(S_{0} D_{r}\right)^{-1} A_{0}\left(S_{0} D_{r}\right)-\sum_{j=1}^{N}\left(S_{j} D_{r}\right)^{-1} A_{j}\left(S_{j} D_{r}\right)
$$

has unbounded $(2,1)$ entry as $r \rightarrow \infty$. The conclusion follows.

Determining

$$
\inf \left\|A_{0}-\sum_{j=1}^{N} S_{j}^{-1} A_{j} S_{j}\right\|
$$

is more challenging. Let us first consider two matrices $A, B \in M_{n}$. We have the following.

Proposition 5.2. Let $A, B \in M_{n}$. Then for any unitary similarity invariant norm $\|\cdot\|$

$$
\left\|(\operatorname{tr} A-\operatorname{tr} B) \frac{1}{n} \mathbb{1}\right\| \leq\left\|S^{-1} A S-T^{-1} B T\right\|
$$

for any invertible $S$ and $T$.

Proof. Given two real vectors $x=\left(x_{1}, \ldots, x_{n}\right), y=\left(y_{1}, \ldots, y_{n}\right)$, we say that $x$ is weakly majorized by $y$, denoted by $x \prec_{w} y$ if the sum of the $k$ largest entries of $x$ is not larger than that of $y$ for $k=1, \ldots, n$. By the Ky Fan dominance theorem, if $X=\operatorname{diag}\left(x_{1}, \ldots, x_{n}\right)$ and $Y=\operatorname{diag}\left(y_{1}, \ldots, y_{n}\right)$ are nonnegative matrices such that $\left(x_{1}, \ldots, x_{n}\right) \prec_{w}\left(y_{1}, \ldots, y_{n}\right)$, then $\|X\| \leq\|Y\|$ for any unitarily invariant norm $\|\cdot\|$.

Now, suppose $S^{-1} A S-T^{-1} B T$ has diagonal entries $d_{1}, \ldots, d_{n}$ and singular values $s_{1}, \ldots, s_{n}$. Then

$$
|\operatorname{tr} A-\operatorname{tr} B|=\left|\sum_{j=1}^{n} d_{j}\right| \leq \sum_{j=1}^{n}\left|d_{j}\right| .
$$

Thus,

$$
|\operatorname{tr} A-\operatorname{tr} B|(1, \ldots, 1) / n \prec_{w}\left(\left|d_{1}\right|, \ldots,\left|d_{n}\right|\right) \prec_{w}\left(s_{1}, \ldots, s_{n}\right) .
$$

It follows that

$$
\begin{aligned}
\left\|(\operatorname{tr} A-\operatorname{tr} B) \frac{1}{n} \mathbb{1}_{n}\right\| & \leq \| \operatorname{diag}\left(\left|d_{1}\right|, \ldots,\left|d_{n}\right|\right) \\
& \leq\left\|\operatorname{diag}\left(s_{1}, \ldots, s_{n}\right)\right\|=\left\|S^{-1} A S-T^{-1} B T\right\| .
\end{aligned}
$$

Can we always find invertible $S$ and $T$ such that

$$
\left\|S^{-1} A S-T^{-1} B T\right\|=\left\|(\operatorname{tr} A-\operatorname{tr} B) \frac{1}{n} \mathbb{1}\right\| ?
$$

The answer is no, and we have the following. 
Proposition 5.3. Let $\|\cdot\|$ be a unitarily invariant norm on $M_{n}$. Suppose $A \in M_{n}$ has eigenvalues $a_{1}, \ldots, a_{n}$, and $B=b \mathbb{1}$. Then

$$
\inf \left\{\left\|S^{-1} A S-B\right\|: S \in M_{n} \text { is invertible }\right\}=\left\|\operatorname{diag}\left(a_{1}-b, \ldots, a_{n}-b\right)\right\| .
$$

Proof. Suppose $S^{-1} A S-B$ has eigenvalues $a_{1}-b, \ldots, a_{n}-b$, and singular values $s_{1}, \ldots, s_{n}$. Then the product of the $k$ largest entries of the vector $\left(\left|a_{1}-b\right|, \ldots, \mid a_{n}-\right.$ $b \mid)$ is not larger than $\left(s_{1}, \ldots, s_{n}\right)$ for $k=1, \ldots, n$. It follows that

$$
\left(\left|a_{1}-b\right|, \ldots,\left|a_{n}-b\right|\right) \prec_{w}\left(s_{1}, \ldots, s_{n}\right),
$$

and hence

$$
\left\|\operatorname{diag}\left(\left|a_{1}-b\right|, \ldots,\left|a_{n}-b\right|\right)\right\| \leq\left\|\operatorname{diag}\left(s_{1}, \ldots, s_{n}\right)\right\|=\left\|S^{-1} A S-B\right\| .
$$

Note that there is $S$ such that $S^{-1}(A-B) S$ is in upper triangular Jordan form with diagonal entries $a_{1}-b, \ldots, a_{n}-b$. Let $D_{r}=\operatorname{diag}\left(1, r, \ldots, r^{n-1}\right)$ for $r>0$. Then $\left(S D_{r}\right)^{-1}(A-B)\left(S D_{r}\right) \rightarrow \operatorname{diag}\left(a_{1}-b, \ldots, a_{n}-b\right)$ and $\left\|\left(S D_{r}\right)^{-1}(A-B)\left(S D_{r}\right)\right\| \rightarrow$ $\left\|\operatorname{diag}\left(a_{1}-b, \ldots, a_{n}-b\right)\right\|$ as $r \rightarrow 0$. Hence, the conclusion about the infimum.

From the above result and proof, we see that if $A$ has an eigenvalue $a$ with eigenspace of dimension $p$ and $B$ has an eigenvalue $b$ with eigenspace of dimension $q$ such that $p+q-n=r>0$, then $S^{-1} A S-T^{-1} B T$ has an eigenvalue $a-b$ of multiplicity at least $r$. The question is whether we can write $A=a \mathbb{1}_{r} \oplus A_{1}$ and $B=b \mathbb{1}_{r} \oplus B_{1}$ and show that

$$
\inf \left\|S_{1}^{-1} A_{1} S_{1}-T_{1}^{-1} B_{1} T_{1}\right\|=\left\|\left(\operatorname{tr} A_{1}-\operatorname{tr} B_{1}\right) \frac{1}{n-k} \mathbb{1}_{n-k}\right\| .
$$

It is interesting to note that the following two quantities may be different:

1) $\inf \left\{\left\|S^{-1} A S-T^{-1} B T\right\|: S\right.$ is invertible $\}$.

2) $\inf \left\{\left\|S^{-1} A S-B\right\|: S\right.$ is invertible $\}$.

For example, suppose $A=\operatorname{diag}(2,-1,-1)$ and $B=\left(\begin{array}{lll}0 & 1 & 0 \\ 0 & 0 & 1 \\ 0 & 0 & 0\end{array}\right)$. Then there are invertible $S$ and $T$ such that

$$
S^{-1} A S=\left(\begin{array}{ccc}
0 & 1 & 1 \\
1 & 0 & 1 \\
1 & 1 & 0
\end{array}\right) \quad \text { and } \quad T^{-1} B T=\left(\begin{array}{ccc}
0 & 1 & 1 \\
0 & 0 & 1 \\
0 & 0 & 0
\end{array}\right) .
$$

So, $C=S^{-1} A S-T^{-1} B T$ is a rank two nilpotent. Thus for any $\varepsilon>0$, there is an invertible $R_{\varepsilon}$ such that

$$
R_{\varepsilon}^{-1} C R_{\varepsilon}=\left(\begin{array}{ccc}
0 & \varepsilon & 0 \\
0 & 0 & \varepsilon \\
0 & 0 & 0
\end{array}\right)
$$

As a result,

$$
\left\|R_{\varepsilon}^{-1} S^{-1} A S R_{\varepsilon}-R_{\varepsilon}^{-1} T^{-1} B T R_{\varepsilon}\right\| \rightarrow 0 \quad \text { as } \quad \varepsilon \rightarrow 0
$$

So, the quantity in (1) equals zero. On the other hand, for every invertible $S$, we have

$$
\left\|\left(A-S B S^{-1}\right)\left(S e_{1}\right)\right\|=\left\|A\left(S e_{1}\right)\right\| \geq\left\|S e_{1}\right\| .
$$

Therefore, inf $\left\|A-S B S^{-1}\right\| \geq 1$. So, we see that the quantities in (1) and (2) may be different. 
TABLE 1. Summary of least-squares approximations by matrix orbits and related gradient flows

\begin{tabular}{|c|c|}
\hline type and objective & coupled gradient flows \\
\hline $\begin{array}{l}\text { unitary similarity: } \\
\min _{U \in S U(n)}=\left\|\sum_{j=1}^{N} U_{j} A_{j} U_{j}^{*}-A_{0}\right\|\end{array}$ & $\begin{array}{l}U_{k+1}^{(j)}=\exp \left\{-\alpha_{k}^{(j)}\left[A_{k}^{(j)}, A_{0 j k}^{*}\right]_{s}\right\} U_{k}^{(j)} \\
\quad \text { where } A_{k}^{(j)}:=U_{k}^{(j)} A_{j} U_{k}^{(j)^{*}} \\
\quad \text { and } A_{0 j k}:=A_{0}-\sum_{\substack{\nu=1 \\
\nu \neq j}}^{N} A_{k}^{(\nu)}\end{array}$ \\
\hline $\begin{array}{l}\text { unitary equivalence: } \\
\min _{U, V \in S U(n)}\left\|\sum_{j=1}^{N} U_{j} A_{j} V_{j}-A_{0}\right\|\end{array}$ & $\begin{array}{l}U_{k+1}^{(j)}=\exp \left\{-\alpha_{k}^{(j)}\left(U_{k}^{(j)} A_{j} V_{k}^{(j)} A_{0 j k}^{*}\right)_{s}\right\} U_{k}^{(j)} \\
V_{k+1}^{(j)}=\exp \left\{-\beta_{k}^{(j)}\left(V_{k}^{(j)} A_{0 j k}^{*} U_{k}^{(j)} A_{j}\right)_{s}\right\} V_{k}^{(j)} \\
\quad \text { where } \quad A_{0 j k}:=A_{0}-\sum_{\substack{\nu=1 \\
\nu \neq j}}^{N} U_{k}^{(\nu)} A_{\nu} V_{k}^{(\nu)}\end{array}$ \\
\hline $\begin{array}{l}\text { unitary t-congruence: } \\
\min _{U \in S U(n)}\left\|\sum_{j=1}^{N} U_{j} A_{j} U_{j}^{t}-A_{0}\right\|\end{array}$ & $\begin{array}{l}U_{k+1}^{(j)}=\exp \left\{-\alpha_{k}^{(j)}\left(A_{k}^{(j)} A_{0 j k}^{*}+\left(A_{0 j k}^{*} A_{k}^{(j)}\right)^{t}\right)_{s}\right\} U_{k}^{(j)} \\
\quad \text { where } A_{k}^{(j)}:=U_{k}^{(j)} A_{j} U_{k}^{t(j)} \\
\quad \text { and } A_{0 j k}:=A_{0}-\sum_{\substack{\nu=1 \\
\nu \neq j}}^{N} A_{k}^{(\nu)}\end{array}$ \\
\hline
\end{tabular}

In connection to the above discussion, it is interesting to study the following problem:

1. Determine

$$
\inf \left\{\left\|S^{-1} A S-T B T^{-1}\right\|: S, T \text { are invertible }\right\}
$$

and characterize the matrix pairs $(A, B)$.

2. Determine

$$
\inf \left\{\left\|S^{-1} A S-B\right\|: S \text { is invertible }\right\}
$$

and characterize the matrix pairs $(A, B)$ attaining the infimum if they exist.

\section{Conclusions}

We have treated the least-squares approximation problems by elements on the sum of various matrix orbits including unitary similarity, equivalence and congruence. Special attention has been paid to sums of unitary similarity orbits of a Hermitian $A$ and a skew-Hermitian $B$, where theoretical results have been obtained and shown to be consistent with numerical findings. Furthermore, stimulated by numerical experiments, new results on unitary equivalence orbits have been established.

A general framework based on the gradient flows on matrix orbits arising from Lie group actions has been developed to study the proposed problems. The gradient flows devised to this end extend the existing toolbox (see e.g. 2, 10, 32] ) by referring to sums of matrix orbits as summerized in Table 1 . This general approach can be 
used to treat many problems in theory and applications. For instance, flows on such sums of unitary similarity orbits can also be envisaged as flows on unitaries taking a block-diagonal form, and hence they relate to relative $C$ numerical ranges, where the group action is restricted to a compact subgroup $\mathbf{K} \subsetneq S U(n)$ of the full unitary group 31. Needless to say, the flows presented in this work may be restricted from sums of group orbits to sums of subgroup orbits by straightforward projection techniques 32, e.g., in order to address problems in quantum information or in completely positive maps (quantum channels).

Finally, the first explorative results on matrix orbits under noncompact group actions invite follow-up studies.

\section{FURTHER RESEARCH}

In order to avoid the search in our algorithms is terminating in local extrema, one has to choose a sufficiently large set of random unitaries distributed according to the Haar measure. Actually, one knows there are commutation properties at the critical points. It would be nice to find a more efficient method to choose starting points for the search, and prove theorems ensuring that the absolute minimum will be reached from one of these starting points using our algorithms.

Our discussion is focused on orbits of matrices under actions of compact groups. We can consider other orbits under actions of noncompact groups. To continue the list from the introductary section, here are some examples for $S, T \in S L(n, \mathbf{C})$ :

(e) the general similarity orbit of a square matrix $A$ is the set of matrices of the form $S A S^{-1}$,

(f) the equivalence orbit of a rectangular matrix $A$ is the set of matrices of the form $S A T$,

(g) the $*$-congruence orbit of a complex square matrix $A$ is the set of matrices of the form $S A S^{*}$,

(h) the $t$-congruence orbit of a square matrix $A$ is the set of matrices of the form $S A S^{t}$.

However, the fact that $G L(n, \mathbf{C})$ and $S L(n, \mathbf{C})$ are just locally compact entails that in either case there is no bi-invariant Riemannian metric on the tangent spaces, but only left- or right-invariant metrics. Hence, the Hilbert-Schmidt scalar product $\langle B \mid A\rangle=\operatorname{tr}\left\{B^{*} A\right\}$ has to be treated with care, in particular, since we are interested in the complex domain. Moreover, while in compact Lie groups the exponential map is surjective and geodesic [1, in locally compact Lie groups, it is generically neither surjective nor geodesic. It is for these reasons that devising gradient flows in locally compact Lie groups is the subject of a follow-up study.

\section{ACKNOWLEDGMENT}

We wish to thank an anonymous referee for useful questions concerning uniqueness of extrema as well as regarding different random distributions of matrix elements.

\section{REFERENCES}

1. A. Arvanitoyeorgos, An Introduction to Lie Groups and the Geometry of Homogeneous Spaces, Amer. Math. Soc., Providence, RI, 2003. [especially p. 125 ff]. MR2011126 (2004i:53059)

2. A. Bloch, Ed., Hamiltonian and Gradient Flows, Algorithms and Control, Fields Institute Communications, Amer. Math. Soc., Providence, RI, 1994. MR.1297981 (95d:00025) 
3. R.W. Brockett, Dynamical Systems that Sort Lists, Diagonalise Matrices, and Solve Linear Programming Problems, In Proc. IEEE Decision Control, 1988, Austin, Texas, pages 779-803, 1988; reproduced in: Lin. Alg. Appl. 146 (1991), 79-91. MR.1083465 (92j:90043)

4. A.S. Buch, The Saturation Conjecture (after A. Knutson and T. Tao) with an Appendix by William Fulton, Enseign. Math. 46 (2000), 43-60. MR.1769536 (2001g:05105)

5. C.M. Cheng, R.A. Horn, and C.K. Li, Inequalities and Equalities for the Cartesian Decomposition, Lin. Alg. Appl. 341 (2002), 219-237. MR.1873621 (2002m:15012)

6. M.D. Choi, Completely Positive Linear Maps on Complex Matrices, Lin. Alg. Appl. 10 (1975), 285-290. MR0376726 (51:12901)

7. M.D. Choi and P.Y. Wu, Convex Combinations of Projections, Lin. Alg. Appl. 136 (1990), 25-42. MR 1061537 (91e:15018)

8. M.D. Choi and P.Y. Wu, Finite-Rank Perturbations of Positive Operators and Isometries, Studia Math. 173 (2006), 73-79. MR2204463 (2006i:47026)

9. M. Christandl, A Quantum Information-Theoretic Proof of the Relation between Horn's Problem and the Littlewood-Richardson Coefficients, Lect. Notes Comput. Sci. 5028 (2008), 120128. MR2507008

10. M. T. Chu, F. Diele, and I. Sgura, Gradient Flow Methods for Matrix Completion with Prescribed Eigenvalues, Lin. Alg. Appl., 379 (2004), 85-112 . MR2039299 (2005b:65041)

11. J. Day, W. So and R.C. Thompson, The Spectrum of a Hermitian Matrix Sum, Lin. Alg. Appl. 280 (1998), 289-332. MR1644987 (99f:15009)

12. K. Fan and G. Pall, Embedding Conditions for Hermitian and Normal Matrices, Canad. J. Math. 9 (1957), 298-304. MR0085216 (19:6e)

13. W. Fulton, Eigenvalues, Invariant Factors, Highest Weights, and Schubert Calculus, Bull. Amer. Math. Soc. 37 (2000), 209-249. MR.1754641 (2001g:15023)

14. S. J. Glaser, T. Schulte-Herbrüggen, M. Sieveking, O. Schedletzky, N. C. Nielsen, O. W. Sørensen, and C. Griesinger, Unitary Control in Quantum Ensembles: Maximizing Signal Intensity in Coherent Spectroscopy, Science 280 (1998), 421-424.

15. U. Helmke, K. Hüper, J. B. Moore, and T. Schulte-Herbrüggen, Gradient Flows Computing the C-Numerical Range with Applications in NMR Spectroscopy, J. Global Optim. 23 (2002), 283-308. MR1923048 (2003g:90127)

16. U. Helmke and J. B. Moore, Optimisation and Dynamical Systems, Springer, Berlin, 1994.

17. A. Horn, Eigenvalues of Sums of Hermitian Matrices, Pacific J. Math. 12 (1962), 225-241. MR0140521 (25:3941)

18. R.A. Horn and C.R. Johnson, Matrix Analysis, Cambridge University Press, New York, 1985. MR $832183(87 \mathrm{e}: 15001)$

19. A.A. Klyachko, Stable Bundles, Representation Theory and Hermitian Operators, Selecta Math. (N.S.) 4 (1998), 419-445. MR.1654578(2000b:14054)

20. K. Kraus, States, Effects, and Operations, Lecture Notes in Physics, Vol. 190, Springer, Berlin, 1983. MR.725167 (86j:81008)

21. A. Knutson and T. Tao, The Honeycomb Model of $\mathrm{GL}_{n}(\mathbb{C})$ Tensor Products. I. Proof of the Saturation Conjecture, J. Amer. Math. Soc. 12 (1999), 1055-1090. MR.1671451 (2000c:20066)

22. A. Knutson and T. Tao, Honeycombs and Sums of Hermitian Matrices, Notices Amer. Math. Soc. 48 (2001), 175-185. MR1811121 (2002g:15020)

23. T.G. Lei, Congruence Numerical Ranges and Their Radii, Lin. Multilin. Alg. 43 (1998), 411-427. MR 1616480 (98k:15039)

24. C.K. Li, C-Numerical Ranges and C-Numerical Radii, Lin. Multilin. Alg. 37 (1994), 51-82. MR.1313758 (95k:15039)

25. C. K. Li and Y. T. Poon, Diagonals and Partial Diagonals of Sum of Matrices, Canadian J. Math. 54 (2002), 571-594. MR.1900764 (2003a:15017)

26. C.K. Li and N.K. Tsing, On Unitarily Invariant Norms and Related Results, Lin. Multilin. Alg. 20 (1987), 107-119. MR878289 (88c:15015)

27. E. Marques de Sá, On the Inertia of Sums of Hermitian Matrices, Lin. Alg. Appl. 37 (1981), 143-159. MR636216 (84h:15026c)

28. A.W. Marshall and I. Olkin, Inequalities: Theory of Majorization and its Applications, Mathematics in Science and Engineering, Vol. 143. Academic Press, New York-London, 1979. MR552278 (81b:00002)

29. F. Mezzadri, How to Generate Random Matrices from the Classical Compact Groups, Notices Amer. Math. Soc. 54 (2007), 592-604. MR2311982(2008e:22005) 
30. L. Mirsky, Symmetric Gauge Functions and Unitarily Invariant Norms, Quart. J. Math. Oxford 11 (1960), 50-59. MR0114821 (22:5639)

31. T. Schulte-Herbrüggen, G. Dirr, U. Helmke and S. Glaser, The Significance of the C-Numerical Range and the Local C-Numerical Range in Quantum Control and Quantum Information, Lin. Multilin. Alg. 56 (2008), 3-26. MR2378299 (2008m:47005)

32. T. Schulte-Herbrüggen, G. Dirr, U. Helmke and S. Glaser, Gradient Flows for Optimisation in Quantum Information and Quantum Dynamics: Foundations and Applications, Rev. Math. Phys. 22 (2010), 597-667. MR2665760

33. H. Shapiro, A Survey of Canonical Forms and Invariants for Unitary Similarity, Lin. Alg. Appl. 147 (1991), 101-167. MR.1088662 (92d:15013)

34. L. O'Shea and R. Sjamaar, Moment Maps and Riemannian Symmetric Pairs, Math. Ann. 317 (2000), 415-457. MR1776111 (2001g:53146)

35. R.C. Thompson and L.J. Freede, On the Eigenvalues of Sums of Hermitian Matrices, Lin. Alg. Appl. 4 (1971) 369-376. MR0288132 (44:5330)

36. R.C. Thompson and L.J. Freede, On the Eigenvalues of Sums of Hermitian Matrices, II., Aequationes Math. 5 (1970), 103-115. MR0292866 (45:1948)

37. R.C. Thompson, Singular Values and Diagonal Elements of Complex Symmetric Matrices, Lin. Alg. Appl. 26 (1979), 65-106. MR535680 (80f:15011)

38. R.C. Thompson, The Congruence Numerical Range, Lin. Multilin. Alg. 8 (1979/80), 197-206. MR:560560 (81b:15026)

39. H. Weyl, Das asymptotische Verteilungsgesetz der Eigenwerte linearer partieller Differentialgleichungen, Math. Ann. 71 (1912), 441-479 MR:1511670

Department of Mathematics, College of William and Mary, Williamsburg, Virginia 23187

E-mail address: ckli@math.wm.edu

Department of Mathematics, Iowa State University, Ames, Iowa 50051

E-mail address: ytpoon@iastate.edu

Department of Chemistry, Technical University of Munich, D-85747, Garching, GerMANY.

E-mail address: tosh@ch.tum.de 